\title{
BMJ Open Implementation of interventions targeting the uptake of genetic testing services for breast cancer risk: protocol for a systematic review
}

\author{
Subash Thapa (D) , ${ }^{1}$ Anja Leppin, ${ }^{2}$ Rikke Kristensen, ${ }^{2}$ Mette Just Bonde, ${ }^{2}$ \\ Arja R Aro $^{2}$
}

To cite: Thapa S, Leppin A, Kristensen $\mathrm{R}$, et al. Implementation of interventions targeting the uptake of genetic testing services for breast cancer risk: protocol for a systematic review. BMJ Open 2020;10:e031727. doi:10.1136/ bmjopen-2019-031727

- Prepublication history and additional material for this paper are available online. To view these files, please visit the journal online (http://dx.doi org/10.1136/bmjopen-2019031727).

Received 16 May 2019 Revised 14 May 2020 Accepted 21 May 2020
A) Check for updates

(C) Author(s) (or their employer(s)) 2020. Re-use permitted under CC BY-NC. No commercial re-use. See rights and permissions. Published by BMJ.

${ }^{1}$ Research Unit of General Practice, University of Southern Denmark, J.B. Winsløws Vej 9 , 5000 Odense, Denmark ${ }^{2}$ Unit for Health Promotion Research, University of Southern Denmark, Niels Bohrs Vej 9-10, 6700 Esbjerg, Denmark

Correspondence to

Dr Subash Thapa;

sthapa@health.sdu.dk

\section{ABSTRACT}

Introduction The timely identification of breast cancerrelated pathogenic variants can help to identify the risk of potential disease development and determine healthcare choices. However, the uptake rate of genetic testing services for breast cancer risk remains low in many countries. Interventions targeting the uptake of these services among individuals potentially at risk for inherited breast cancer are often complex and have multiple components, and are therefore difficult to implement, replicate and disseminate to new contexts. Our aim is to systematically review studies targeting the uptake of genetic testing services for breast cancer risk and critically assess the quality of implementation outcomes and the reporting of intervention descriptions.

Methods and analysis PubMed, CINAHL, PsycINFO, Embase, Cochrane Library and all Campbell Coordinating Group databases will be searched for intervention studies that target individuals' participation in breast cancer genetic testing programmes. Papers published in English within the time period from January 2005 until October 2019 will be considered for inclusion. Titles, abstracts and full papers will be screened for eligibility by two pairs of reviewers independently. For data analysis and synthesis, study-level and intervention-level characteristics will be abstracted. We will present all implementation outcomes that are mentioned in each of the studies and register the number of studies that do not at all look at or report implementation outcomes. The quality of implementation will be checked using a 5-point rubric item, and the quality and completeness of reporting of intervention description will be evaluated using the 12-item Template for Intervention Description and Replication (TIDieR).

Ethics and dissemination Ethical approval is not required to conduct this review. Review findings will be disseminated to academic and non-specialist audiences via peer-reviewed academic journals and presented at appropriate conferences, workshops and meetings to policymakers, practitioners and organisations that work with our population of interest.

PROSPERO registration number CRD42018105732.

\section{INTRODUCTION}

Inherited pathogenic variants in high/ moderate penetrance breast cancer
Strengths and limitations of this study

- This systematic review will be the first to critically assess the level and quality of implementation of interventions targeting the uptake of breast cancer genetic testing.

- This review will be the first to document the level of reporting of implementation outcomes in such intervention studies and to assess the completeness of intervention descriptions.

- The implementation of interventions targeting genetic testing uptake for breast cancer risk can be strongly influenced by contextual factors. Exploring the relevance and effects of such factors is not within the scope of this review.

- Description and characterisation of implementation parameters may be limited by insufficient reporting in primary studies.

susceptibility genes, such as BRCA1, BRCA2, TP53, PTEN, PALB2, CHEK2, are estimated to play a key role in about $5 \%$ to $10 \%$ of all breast cancers. ${ }^{1}{ }^{2}$ Women with pathogenic variants in the high penetrance breast tumour suppressor genes BRCA1 and BRCA2 are estimated to have a $40 \%$ to $70 \%$ lifetime risk of breast cancer and a $20 \%$ to $55 \%$ risk of ovarian cancer as compared with a $12 \%$ lifetime risk of breast cancer and a $1 \%$ to $2 \%$ risk of ovarian cancer in those without pathogenic variants. ${ }^{3}$ Timely identification of germline breast cancer risk enables close monitoring for potential disease development as well as choice of preventive treatments and lifestyle changes. ${ }^{4-7}$ Further, knowledge about hereditary pathogenic variants is relevant since mutated genes can be passed on to the next generation, in turn increasing risk for breast, ovarian, pancreatic and prostate cancer in sons or daughters or both.

While it has been estimated that 1:800 to 1:300 women in the general population carry 
a BRCA $1 / 2$ mutation, amounting to an approximate prevalence rate of $0.13 \%$ to $0.3 \%$, rates of $2.5 \%$ have been found in individuals of Ashkenazi-Jewish descent. ${ }^{19-11}$ Differences also occur within the subgroup of breast cancer patients. A recent systematic review reported a prevalence for BRCA germline mutations of about $3 \%$ in breast cancer patient groups unselected for family history, sex, age, ethnicity, breast cancer stage or hormone receptor status while rates between $9.3 \%$ and $15.4 \%$ were found in subgroups with triple-negative breast cancer. ${ }^{10}$

Testing for pathogenic variants in breast cancer susceptibility genes is therefore specifically targeted towards those at high risk. Weighing the evidence, the US Preventive Services Task Force has recently concluded 'that the net benefit of [....] testing for BRCA1/2 mutations, and use of risk-reducing interventions outweighs the harms in women whose family or personal history is associated with an increased risk for potentially harmful mutations in the BRCA $1 / 2$ genes'. ${ }^{1}$

Individuals without a diagnosis of cancer who have a family history of cancer, should be given the opportunity to discuss this history with their physician to decide if a formal assessment and genetic testing to determine their risk of breast cancer is indicated. Those who already have breast cancer may be referred to genetic testing in order to determine disease aetiology, the risk for a second primary cancer and to inform treatment, as well as to clarify breast cancer risk for their children and/or siblings. ${ }^{12}$

A recent review of international guidelines and recommendations for BRCA-related breast cancer genetic screening, diagnosis, counselling and treatment found agreement between guidelines in terms of key features, such as the main indicators of a 'high-risk family constellation' or a generally strong endorsement of counselling before and after testing. ${ }^{9}$ However, there also were differences regarding specifications of individual clinical and family history risk criteria which might result in different levels of inclusivity. ${ }^{9}$ In general though, the authors noted a growing trend towards broader inclusion based on concerns that stricter eligibility criteria might exclude too many individuals who actually harbour pathogenic gene alterations. $^{913}$ In line with this, the recent Consensus Guideline on Genetic Testing for Hereditary Breast Cancer by the American Society of Breast Surgeons (2019) has suggested offering genetic testing to all patients with a personal breast cancer history. ${ }^{14}$

Concerns have also been voiced with regard to uptake/ receipt of testing among those who receive the offer to be tested. Ropka et al (2006), in a systematic review, found that rates ranged widely from $25 \%$ to $96 \%$ across different population groups and contexts, ${ }^{15}$ and there is further evidence that low uptake rates are more likely to occur in population groups with lower education, lower income and/or in those with an ethnic minority background. ${ }^{16-18}$ Besides lack of insurance, main drivers for lower uptake in these segments may be lower awareness about testing options, lack of physician referrals, differential access to cancer genetics experts, testing sites and consulting, as well as language barriers. ${ }^{16} 19$ However, it also needs to be noted that a direct comparison of findings is difficult because of varying definitions of 'uptake'. Some studies investigated hypothetical behaviour, that is, intention to participate, while others focussed on actual participation in testing, either using delivery of blood/saliva samples or actual receipt of the result/attendance at a post-test counselling session as criterion. ${ }^{20} 21$

The decision whether and when to take a single gene or multigene panel test, disclosing the presence of pathological variants in breast cancer susceptibility genes, is a challenging process for many. A positive test result might lead to adverse psychological effects (eg, anxiety/worry), potential (felt and/or real) social stigma and discrimination, and it will require complex decisions on different levels. ${ }^{22}{ }^{23}$ Which preventive treatment should be chosen? Regular monitoring, life-style changes or early preventive surgery, such as mastectomy or salpingo-oophorectomy? ${ }^{24}$ And should the result be disclosed to family members who then themselves might face the same emotional consequences and decisional conflicts?

Different types of interventions exist to help people make an informed decision about genetic testing. ${ }^{525} 26$ It is generally recommended that the actual testing process is preceded by a general pre-test counselling session with a specialist and that results are communicated in another counselling session where implications and treatment options can be discussed. ${ }^{9}$ 27-29 Standard offers include specific risk-assessment ${ }^{30}$ and counselling interventions, which can be delivered in different formats such as oneto-one, ${ }^{31}{ }^{32}$ group $^{20}$ and peer-led counselling. ${ }^{21}$ There are also broader education approaches ${ }^{33} 34$ or tailored decision aid interventions. Such interventions are specifically aimed at facilitating understanding and decision-making and include fact-based, written or verbal information on hereditary breast cancer, breast cancer risk of mutation carriers, the general benefits and risks of genetic testing, as well as decision options. ${ }^{33} 34$ Evaluations have so far generated discrepant findings. ${ }^{35} 36$ Bowen et al (2002) ${ }^{20}$ and Schwartz et al (2001) ${ }^{33}$ found that counselling interventions can have a small effect on changing intentions for breast cancer genetic testing. Brain et al (2005), ${ }^{37}$ Helmes et al (2006) ${ }^{31}$ and Bowen (2010), ${ }^{32}$ on the other hand, reported that although face-to-face and telephone counselling interventions changed women's level of knowledge as well as perceived cancer risk and cancer worry, neither of the interventions led to significant changes in intention to pursue genetic testing for breast cancer risk. Venna (2007) ${ }^{21}$ and Hall et al (2009) ${ }^{34}$ also reported that educational interventions had no effect on intentions to participate in cancer genetic testing.

Previous efforts to review interventions targeting breast cancer genetic testing have, however, been limited to explaining uptake rates and predictors of testing decisions, while none of the reviews have focussed on how these interventions have been implemented and whether the implementation strategies used have been effective. ${ }^{152638}$ This is also emphasised by Forbes et al (2019) 
who, in their review about BRCA-testing guidelines, outlined evidence gaps, among them a lack of knowledge about 'optimum models for service delivery and organisation'. 9

Thus, when interventions fail, it often remains unclear whether it was the intervention approach itself which was ineffective (programme failure) or whether the programme never was sufficiently implemented in the first place (implementation failure). Implementation of an intervention relates to how and to which extent an intervention is being delivered or carried out; and implementation success or failure is defined in regard to the degree to which the actual implementation process meets the conceptual definitions of implementation outcomes, such as acceptability, appropriateness, adoption, costs, feasibility, fidelity, penetration and sustainability, as defined by Proctor et al (2011). ${ }^{39} 40$ Knowledge about how informational interventions targeting breast cancer-related genetic testing are implemented could explain why effects fail to occur or remain suboptimal. Moreover, assessing, comparing and analysing implementation outcomes will increase understanding of implementation processes, enable studies about comparative effectiveness of different implementation strategies and enhance efficiency in implementation research. ${ }^{40}$ Such evidence has the potential to inform policymakers and programme managers to make decisions about investing in high-quality implementation of genetic screening and testing programmes for breast cancer risk.

\section{Aim of the review}

The aim of this paper is to systematically review studies targeting the uptake of genetic testing services for breast cancer risk and critically assess the quality of programme implementation and the reporting of implementation outcomes. Specifically, we will:

a. document the general characteristics of included studies and the interventions investigated;

b. document the implementation strategies reported in each of the intervention studies;

c. examine the quality of intervention implementation in terms of acceptability, appropriateness, adoption, costs, feasibility, fidelity, penetration and sustainability; and

d. evaluate the completeness and quality of the reported information about implementation.

\section{METHODS}

This will be a systematic review documenting the evidence concerning implementation of interventions that target the uptake of genetic testing services for breast cancer risk. We will follow the Preferred Reporting Items for Systematic Reviews and Meta-Analysis (PRISMA) guidelines. ${ }^{41}$

We will include intervention studies targeting uptake of predictive genetic testing services (single gene or multigene panel testing) as well as decision-making about using such services to detect pathogenic variants in breast cancer predisposition genes of moderate (PALB2, ATM, CHEK2) and high penetrance (BRCA1, BRCA2, TP53, PTEN, CDH1, STK11). Specifically, the review will include the studies targeting individuals without a breast cancer diagnosis but a family history of breast cancer to assess their risk for future disease manifestation. ${ }^{42}{ }^{43}$ The definition of 'family history of breast cancer' is based on the criteria documented in the international guideline review by Forbes et al $(2019)^{9}$ or the European School of Oncology-European Society of Medical Oncology (ESOESMO) Consensus Guidelines (ABC 4$)^{42}$ or two newer US National Comprehensive Cancer Network (NCCN) Guidelines $(2018,2019) .{ }^{28}{ }^{44}$ Further, studies reporting on interventions for individuals from an Ashkenazi-Jewish ancestry due to their higher risk of having pathogenic variants in BRCA1/2 genes will be included. The third major group of interest for this review are individuals diagnosed with breast cancer who are referred by their healthcare provider for predictive genetic counselling/ testing in order to identify a potential breast cancer risk for their family members due to a pathogenic variant in the index patient's breast cancer susceptibility genes. ${ }^{43} 45$

Not included will be intervention studies which focus exclusively on men as a primary target group and studies which specifically target genetic test uptake with the goal of guiding the choice of treatment for breast cancer patients or understanding disease progression or patients' response to treatments. ${ }^{43}$ Also, intervention studies which only target healthcare professionals (eg, geneticists, genetic counsellors) or other relevant occupational populations will be excluded. For a more specific definition of inclusion criteria see the section on 'study selection' below.

\section{Type of intervention}

We will include intervention studies targeting the uptake of breast cancer genetic testing service (registered/ self-reported) or studies seeking to influence decisionmaking to pursue breast cancer genetic testing. Examples of such interventions include: one-to-one (face-to-face or via telephone) genetic counselling; ${ }^{31} 32$ group counselling: ${ }^{20}$ print materials-based education interventions; $; 334$ computer-based education interventions $;{ }^{30}$ peer-led interventions; ${ }^{21}$ and tailored decision aid interventions. ${ }^{35}$

\section{Outcomes}

Only intervention studies targeting decision-making about and/or actual uptake (registered/self-reported) of breast cancer genetic testing service will be included in the review.

\section{Study types}

This review will include scientific research based on qualitative studies as well as quantitative designs, mainly randomised controlled trials and controlled before and after studies, which investigate interventions targeting decision-making about and/or actual uptake (registered/ self-reported) of breast cancer genetic testing service. 
There will be no restrictions for inclusion of studies based on number of interventions and control groups, length of follow-up or study quality.

Unpublished, non-peer reviewed, theoretical and methodological research papers as well as editorials and opinion pieces will be excluded.

Only papers that have been published in English within the time period from January 2005 until October 2019 will be considered for inclusion. The rationale for this time period is based on the publication of the highly influential Guidelines by the US Preventive Services Task Force in 2005, which extended eligibility criteria for use of $B R C A 1 / 2$ genetic testing and explicitly advocated the use of genetic testing for at-risk women. ${ }^{45}$ Since then the cost for genetic testing has significantly decreased, genetic testing has become more accessible and more interventions targeting the uptake of genetic testing services for breast cancer in at-risk women have been implemented. ${ }^{46}$

\section{Search strategy}

We will perform a systematic electronic database search in the following databases: PubMed, CINAHL, PsycINFO, Embase, Cochrane Library and all Campbell Coordinating Group databases. Further, the reference list of primary studies will be searched for additional studies. We have developed a list of terms for each concept and key search terms, which is illustrated in box 1 . The finalised search strategy for Embase is provided in online supplementary appendix 1 .

\section{Study selection}

A two-step process will be used to screen studies for inclusion. At first, four reviewers in pairs of two will independently screen all titles, abstracts and full-text articles. Studies will pass the title/abstract screening stage if the title or abstract mentions any intervention/s targeting genetic testing uptake for breast cancer risk and if the intervention (or component of the intervention) is targeted to one or more of the following subgroups:

1. Individuals without a diagnosis of breast cancer

a. and a family history of breast cancer and/or other relevant cancers as defined by any of the respective criteria constellations listed in the review of international guidelines for screening and diagnosis by Forbes et al (2019), ${ }^{9}$ or by the newer US NCCN Guidelines $(2018,2019)^{28} 44$ or the European ESOESMO Consensus Guidelines (ABC 4$)^{42}$

b. and a personal history of a non-breast cancer known to be related to breast cancer based on criteria listed by the above-mentioned guideline review ${ }^{9}$ or the newer guidelines ${ }^{28} 4244$

c. who are of Ashkenazi-Jewish descent

d. who have a known pathogenic variant in a breast cancer susceptibility gene in the family ${ }^{9} 24244$

2. Individuals with a diagnosis of breast cancer

a. with certain specific characteristics of the cancer (for instance triple negative breast cancer, young age at diagnosis) as defined by the guidelines re-

\section{Box 1 Key search terms}

(Breast neoplasm OR breast tumour OR breast carcinoma OR mammary neoplasms OR mammary cancer OR malignant neoplasm of breast OR malignant tumour of breast $\mathrm{OR}$ cancer of breast $\mathrm{OR}$ breast cancer) AND

(intervention OR programs OR counselling OR provider recommendation OR pamphlet OR brochure OR communication OR training OR peer education $\mathrm{OR}$ health education $\mathrm{OR}$ health information $\mathrm{OR}$ visual information OR group discussion OR media advertisement OR public service announcement OR message OR patient education)

AND

(gene-test $\mathrm{OR}$ genetic evaluation $\mathrm{OR}$ genetic test* $\mathrm{OR}$ genomic test $\mathrm{OR}$ genomic technology OR health testing OR multiphasic test OR pharmacogenetic test OR direct-to-consumer screening OR consumer screening OR genetic screen* OR genomic screen* OR mass screening OR mandatory testing $\mathrm{OR}$ whole-body imaging $\mathrm{OR}$ whole-body screening OR genetic exam* OR genetic assess* OR genetic evaluate* $O R$ direct DNA sequencing OR sanger sequencing OR next-generation sequencing OR BRCA1 testing OR BRCA2 testing OR TP53 testing OR PTEN testing OR CDH1 testing OR STK11 testing OR PALB2 testing OR ATM testing OR CHEK2 testing)

AND

(acceptability OR acceptable OR advantage OR credibility OR appropriateness $\mathrm{OR}$ appropriate $\mathrm{OR}$ approved $\mathrm{OR}$ approval $\mathrm{OR}$ enacted $\mathrm{OR}$ endorsed OR endorsement OR maintained OR maintenance OR embraced $O R$ selected $O R$ selection $O R$ chosen $O R$ choice $O R$ suitable $O R$ suitability OR suited OR apt OR useful OR usefulness OR adoption OR adopted $\mathrm{OR}$ uptake $\mathrm{OR}$ utilisation $\mathrm{OR}$ intention $\mathrm{OR}$ cost $\mathrm{OR}$ economic* OR expense OR expensive OR expenditure OR expenditures OR pay OR paid $O R$ price $O R$ budget $O R$ feasibility $O R$ feasible $O R$ fit $O R$ achievable $O R$ advantageous $O R$ attainable $O R$ practicable $O R$ practical $O R$ viable OR workable OR worthwhile OR fidelity OR adherent OR adherence OR accuracy $\mathrm{OR}$ accurate $\mathrm{OR}$ precision $\mathrm{OR}$ precise $\mathrm{OR}$ uniform $\mathrm{OR}$ uniformity $\mathrm{OR}$ exact $\mathrm{OR}$ close $\mathrm{OR}$ penetration $\mathrm{OR}$ penetrated $\mathrm{OR}$ diffusion $\mathrm{OR}$ sustainability $\mathrm{OR}$ sustainable $\mathrm{OR}$ sustained $\mathrm{OR}$ implementation science OR implementation research OR dissemination research OR implementation outcome OR implementation outcomes)

viewed by Forbes et al $(2019)^{9}$ or the newer guidelines ${ }^{28} 4244$

b. with a known pathogenic variant in a breast cancer susceptibility gene in the family ${ }^{9} 8424$

c. with a family history of breast cancer and/or other relevant cancers, as defined by the guidelines reviewed by Forbes et al $(2019)^{9}$ or the newer guidelines ${ }^{28} 4244$

d. who are of Ashkenazi-Jewish descent

Studies will pass the full-text screening stage if the eligibility criteria above (in terms of population, intervention, outcome and study type) are met. The reviewers will note the reasons for excluding studies during full-text review. Disagreements will be resolved through discussion until a consensus is reached. The web-based reference programme Covidence will be applied as an organising instrument throughout the assessment process to import, include or exclude relevant studies, eliminate duplicate findings and finally develop a PRISMA flow diagram. This diagram will document the process of selection, as well as the numbers of records and full-text papers identified at 
each stage. Given the focus of this review on the implementation process, we will not assess the methodological quality of studies.

\section{Data extraction}

The data extraction process will include the collection of information at two levels: study level and intervention level.

At the study level, we will collect information regarding the research question; methods and study type; implementation research framework used; time of data collection; study population; implementation outcomes reported; study limitations and conclusion. Among the included studies, we will categorise the implementation studies according to three types of hybrid effectivenessimplementation designs: type 1 (studies testing a clinical intervention and exploring implementation-related factors), type 2 (studies testing a clinical intervention and also testing an explicitly described implementation strategy) or type 3 (studies testing an explicitly described implementation strategy and observing/collecting clinical outcomes data). ${ }^{47}$ Implementation strategies can be defined as methods or techniques used to enhance the adoption, implementation and sustainability of a clinical programme or practice', and might involve single or multiple components. ${ }^{47} 48$ We will also note the stage of intervention implementation for each study as 'pilot/ once-off', 'scaling up', 'implemented and sustained at scale' or 'undergoing de-implementation'.

Implementation outcomes will be reported for acceptability, adoption, appropriateness, cost, feasibility, fidelity, penetration and sustainability, as defined by Proctor $e t$ al (see table 1) ${ }^{40}$ We will register the implementation outcomes that are reported in each of the studies as well as the number of studies that do not consider or report any implementation outcomes. To determine whether the included studies tested for implementation quality, we will use the 5-item rubric developed by Kemp et al (2019).$^{49}$ The five items relate to: (a) whether the authors included any implementation outcomes in the study objectives; (b) whether they specified any hypothesis or conceptual model for implementation outcomes; (c) whether they comprehensively described measurement methods for implementation outcomes; (d) whether they used validated measures for implementation outcomes; and (e) whether they reported the sample sizes for implementation outcomes.

At the intervention level, we will collect information about intervention types, intervention descriptions, intervention strategies, countries and target populations. The Template for Intervention Description and Replication (TIDieR) will be used to evaluate the completeness of intervention description and specification (see online supplementary appendix 2). ${ }^{50}$ Each item in the TIDieR checklist will be counted as present if any aspect of the item is mentioned, regardless of quality or level of detail. Risk of bias will not be assessed.

Four reviewers by two pairs will independently pilot a structured data extraction form with four studies. The study and intervention characteristics will be extracted onto a spreadsheet by the main researcher, which will be further checked and verified by three further team members.

\section{Data analysis and synthesis}

The extracted study and intervention characteristics will be summarised and organised in one or more evidence tables. We will use descriptive statistics for categorical

Table 1 Conceptual definition of the implementation outcomes

\section{Implementation}

outcomes

\begin{tabular}{|c|c|c|}
\hline Acceptability & $\begin{array}{l}\text { Perception that the intervention is agreeable, satisfactory or } \\
\text { confers relative advantage }\end{array}$ & Comfort; Advantage; Credibility \\
\hline Appropriateness & Perceived fit or relevance of the intervention in a setting & $\begin{array}{l}\text { Relevance; Perceived fit; Compatibility; } \\
\text { Perceived usefulness; Suitability }\end{array}$ \\
\hline Adoption & $\begin{array}{l}\text { Intention, initial decision or action to try to employ a new } \\
\text { intervention }\end{array}$ & Uptake; Utilisation; Intention to try \\
\hline Costs & $\begin{array}{l}\text { Incremental cost of the implementation strategy and the total cost } \\
\text { of implementation including the cost of the intervention }\end{array}$ & Marginal cost; Total cost \\
\hline Feasibility & $\begin{array}{l}\text { The extent to which an intervention can be carried out in a setting } \\
\text { or organisation }\end{array}$ & Practicality; Actual fit; Utility; Trialability \\
\hline Fidelity & $\begin{array}{l}\text { The degree to which an intervention is implemented as it was } \\
\text { designed in an original protocol, plan or policy }\end{array}$ & $\begin{array}{l}\text { Adherence; Integrity; Quality of } \\
\text { programme delivery; Intensity or dosage } \\
\text { of delivery }\end{array}$ \\
\hline
\end{tabular}


variables and means and SD for continuous variables. An implementation outcome score will be calculated for each study by summing the number of rubric items present and dividing by the total number of applicable items, that is, the total score of 5 . A TIDieR specification score out of 12 will be calculated for each intervention by summing the number of checklist items reported across studies of the same intervention divided by the total number of applicable criteria, that is, the total score of 12.

\section{Ethics and dissemination}

Ethical approval is not required to conduct this review. Results of this study will be disseminated to academic and non-specialist audiences through peer-reviewed publications and presented at appropriate conferences, workshops and meetings to policymakers, practitioners and organisations that work with our population of interest. Evidence generated from this review will be used to inform the development of evidence-based quality implementation strategies to target people at risk for inherited breast cancer for their participation in genetic testing programmes.

\section{Patient and public involvement}

Patients and/or the public will not be (directly or indirectly) involved in this study.

\section{DISCUSSION}

The US Preventive Services Task Force recommends that 'women with a personal or family history of breast cancer or who have an ancestry associated with $B R C A 1 / 2$ gene mutations should be assessed by primary care clinicians with an appropriate brief familial risk assessment tool, and women with a positive result on the risk assessment tool should receive genetic counselling, ${ }^{45}$ Given the complexity involved in risk assessment and the challenges for test recipients and their families in understanding the implications of such tests, genetic counselling should be offered both before and after breast cancer genetic testing. ${ }^{51}$ Limitations of the test as well as the benefits and costs/side effects of obtaining this kind of information and of prophylactic measures should be thoroughly explained so that people can make an informed decision regarding whether they actually want to take the test as well as about potential actions and consequences resulting from potential outcomes. ${ }^{43}$

Thus, an effective intervention targeting genetic testing uptake to determine individuals' increased risk of having breast cancer should incorporate various strategies to reach the individuals at-risk, deliver qualified standard genetic counselling and/or broader educational interventions and be conducted with a set of high implementation and ethical standards. ${ }^{52} 53$ We believe our review will be the first to document whether the published studies on interventions to increase participation in breast cancer genetic testing programmes have tested and reported implementation quality and provided relevant intervention descriptions. We hope, the findings of this systematic review will help improve understanding of the challenges and pitfalls of programme implementation or implementation-related research for breast cancer genetic testing. Furthermore, this might help programme managers to identify or develop better implementation strategies to target individuals at risk of hereditary breast cancer.

This review may have some limitations. First, the implementation of interventions targeting genetic testing can be strongly influenced by contextual factors. Exploring the relevance and effects of such factors is not within the scope of this review. Second, description and characterisation of implementation parameters may be limited by insufficient reporting in primary studies. Third, it cannot be excluded that relevant interventions exist, whose primary goal was not related to decision-making about/uptake of genetic testing; such studies will not be captured by this review.

Acknowledgements We would like to thank the two reviewers, namely Charité Ricker, Keck School of Medicine, University of Southern California (USC), Los Angeles, California, USA and W. David Dotson, Office of Public Health Genomics (OPHG), Centers for Disease Control and Prevention (CDC), Atlanta, Georgia, USA for their extraordinarily helpful comments and efforts towards improving our protocol.

Contributors ST, AL and ARA were responsible for conceptualising the protocol and overall design of the review. The initial draft of the protocol was prepared by ST, which was then circulated among all authors for critical revision. All authors (ST, AL, RK, MJB, ARA) helped to evolve the writing of the literature review, framework, data extraction, analysis and synthesis plan and critically revise successive drafts of the protocol. ST, AL, RK and MJB will review the studies for inclusion and document all the important amendments that will be made during the review process.

Funding The authors have not declared a specific grant for this research from any funding agency in the public, commercial or not-for-profit sectors.

Competing interests None declared.

Patient consent for publication Not required.

Provenance and peer review Not commissioned; externally peer reviewed.

Open access This is an open access article distributed in accordance with the Creative Commons Attribution Non Commercial (CC BY-NC 4.0) license, which permits others to distribute, remix, adapt, build upon this work non-commercially, and license their derivative works on different terms, provided the original work is properly cited, appropriate credit is given, any changes made indicated, and the use is non-commercial. See: http://creativecommons.org/licenses/by-nc/4.0/.

Author note The study screening and data extraction process is anticipated to be finished by July 2020 and the final analysis and writing will be finished by November 2020.

ORCID iD

Subash Thapa http://orcid.org/0000-0002-1182-8511

\section{REFERENCES}

1 US Preventive Services Task Force, Owens DK, Davidson KW, et al. Risk assessment, genetic counseling, and genetic testing for BRCArelated cancer: US Preventive Services Task Force recommendation statement. JAMA 2019;322:652-65.

2 Honrado E, Benítez J, Palacios J. The molecular pathology of hereditary breast cancer: genetic testing and therapeutic implications. Mod Pathol 2005;18:1305-20.

3 Antoniou A, Pharoah PDP, Narod S, et al. Average risks of breast and ovarian cancer associated with BRCA1 or BRCA2 mutations detected in case series unselected for family history: a combined analysis of 22 studies. Am J Hum Genet 2003;72:1117-30.

4 Grimmett C, Pickett K, Shepherd J, et al. Systematic review of the empirical investigation of resources to support decision-making 
regarding BRCA1 and BRCA2 genetic testing in women with breast cancer. Patient Educ Couns 2018;101:779-88.

5 Hamilton RJ, Innella NA, Bounds DT. The life course perspective: a guide for genetic counselors. J Genet Couns 2016;25:44-8.

6 Rahman N. Mainstreaming genetic testing of cancer predisposition genes. Clin Med 2014;14:436-9.

7 Burke W, Khoury MJ, Stewart A, et al. The path from genome-based research to population health: development of an international public health genomics network. Genet Med 2006;8:451-8.

8 van Asperen CJ, Brohet RM, Meijers-Heijboer EJ, et al. Cancer risks in BRCA2 families: estimates for sites other than breast and ovary. $J$ Med Genet 2005;42:711-9.

9 Forbes C, Fayter D, de Kock S, et al. A systematic review of international guidelines and recommendations for the genetic screening, diagnosis, genetic counseling, and treatment of $B R C A$ mutated breast cancer. Cancer Manag Res 2019;11:2321-37.

10 Armstrong N, Ryder S, Forbes C, et al. A systematic review of the international prevalence of BRCA mutation in breast cancer. Clin Epidemiol 2019;11:543-61.

11 Paluch-Shimon S, Cardoso F, Sessa C, et al. Prevention and screening in BRCA mutation carriers and other breast/ovarian hereditary cancer syndromes: ESMO clinical practice guidelines for cancer prevention and screening. Ann Oncol 2016;27:v103-10.

12 Petersen GM, testing G. Genetic testing. Hematol Oncol Clin North Am 2000;14:939-52.

13 Metcalfe KA, Poll A, Royer R, et al. Screening for founder mutations in BRCA1 and BRCA2 in unselected Jewish women. J Clin Oncol 2010;28:387-91.

14 Manahan ER, Kuerer HM, Sebastian M, et al. Consensus guidelines on genetic' testing for hereditary breast cancer from the American society of breast surgeons. Ann Surg Oncol 2019;26:3025-31.

15 Ropka ME, Wenzel J, Phillips EK, et al. Uptake rates for breast cancer genetic testing: a systematic review. Cancer Epidemiol Biomarkers Prev 2006;15:840-55.

16 Cragun D, Weidner A, Lewis C, et al. Racial disparities in BRCA testing and cancer risk management across a populationbased sample of young breast cancer survivors. Cancer 2017; 123:2497-505.

17 Jones T, Lockhart JS, Mendelsohn-Victor KE, et al. Use of cancer genetics services in African-American young breast cancer survivors. Am J Prev Med 2016;51:427-36.

18 Hall MJ, Reid JE, Burbidge LA, et al. BRCA1 and BRCA2 mutations in women of different ethnicities undergoing testing for hereditary breast-ovarian cancer. Cancer 2009;115:2222-33.

19 Swink A, Nair A, Hoof P, et al. Barriers to the utilization of genetic testing and genetic counseling in patients with suspected hereditary breast and ovarian cancers. Proc 2019;32:340-4.

20 Bowen DJ, Burke W, Yasui Y, et al. Effects of risk counseling on interest in breast cancer genetic testing for lower risk women. Genet Med 2002;4:359-65.

21 Venne VL, Hamann HA. Successful use of peer educators for sharing genetic information. $J$ Genet Couns 2007;16:515-25.

22 Schroeder D, Conroy SA. Breast cancer genetic testing: more than a medical management tool. Clin J Oncol Nurs 2015;19:603-7.

23 Surbone A. Social and ethical implications of BRCA testing. Ann Oncol 2011;22:i60-6.

24 Klitzman R, Chung W. The process of deciding about prophylactic surgery for breast and ovarian cancer: patient questions, uncertainties, and communication. Am J Med Genet $A$ 2010;152A:52-66.

25 Thiruchelvam PTR, Fisher CS, Leff DR, et al. Pervasive genetic testing. Lancet 2018;391:2089-91.

26 Hilgart JS, Coles B, Iredale R. Cancer genetic risk assessment for individuals at risk of familial breast cancer. Cochrane Database Syst Rev 2012;2:CD003721.

27 Katz SJ, Ward KC, Hamilton AS, et al. Gaps in receipt of clinically indicated genetic counseling after diagnosis of breast cancer. $J$ Clin Oncol 2018;36:1218-24

28 National Comprehensive Cancer Network. NCCN clinical practice guidelines in oncology (NCCN guidelines) genetic/familial high-risk assessment: breast and ovarian. version 3. 2019. 3025 Chemical Road, Plymouth Meeting, Pennsylvania: National Comprehensive Cancer Network, 2019.

29 National Institute for Health and Care Excellence. Familial breast cancer: classification, care and managing breast cancer and related risks in people with a family history of breast cancer: NICE guidelines. National Institute for Health and Care Excellence, 2013.
30 Green MJ, Peterson SK, Baker MW, et al. Effect of a computerbased decision aid on knowledge, perceptions, and intentions about genetic testing for breast cancer susceptibility: a randomized controlled trial. JAMA 2004:292:442-52.

31 Helmes AW, Culver JO, Bowen DJ. Results of a randomized study of telephone versus in-person breast cancer risk counseling. Patient Educ Couns 2006;64:96-103.

32 Bowen DJ, Powers D. Effects of a mail and telephone intervention on breast health behaviors. Health Educ Behav 2010;37:479-89.

33 Schwartz MD, Benkendorf J, Lerman C, et al. Impact of educational print materials on knowledge, attitudes, and interest in BRCA1/ BRCA2: testing among Ashkenazi Jewish women. Cancer 2001;92:932-40.

34 Hall J, Gray S, A'Hern R, et al. Genetic testing for BRCA1: effects of a randomised study of knowledge provision on interest in testing and long term test uptake; implications for the NICE guidelines. Fam Cancer 2009;8:5-13.

35 Wakefield CE, Meiser B, Homewood J, et al. A randomized controlled trial of a decision aid for women considering genetic testing for breast and ovarian cancer risk. Breast Cancer Res Treat 2008;107:289-301.

36 Raffle AE. Information about screening - is it to achieve high uptake or to ensure informed choice? Health Expect 2001;4:92-8.

37 Brain K, Sivell S, Bennert K, et al. An exploratory comparison of genetic counselling protocols for HNPCC predictive testing. Clin Genet 2005;68:255-61.

38 Légaré $\mathrm{F}$, Robitaille $\mathrm{H}$, Gane $\mathrm{C}$, et al. Improving decision making about genetic testing in the clinic: an overview of effective knowledge translation interventions. PLoS One 2016;11:e0150123.

39 Eldh AC, Almost J, DeCorby-Watson K, et al. Clinical interventions, implementation interventions, and the potential greyness in between -A discussion paper. BMC Health Serv Res 2017;17:16.

40 Proctor E, Silmere H, Raghavan R, et al. Outcomes for implementation research: conceptual distinctions, measurement challenges, and research agenda. Adm Policy Ment Health 2011;38:65-76.

41 Moher D, Liberati A, Tetzlaff J, et al. Preferred reporting items for systematic reviews and meta-analyses: the PRISMA statement. $J$ Clin Epidemiol 2009;62:1006-12.

42 Cardoso F, Senkus E, Costa A, et al. 4th ESO-ESMO international consensus guidelines for advanced breast cancer (ABC 4)†. Ann Oncol 2018;29:1634-57.

43 Rutgers E, Balmana J, Beishon M, et al. European breast cancer council manifesto 2018: genetic risk prediction testing in breast cancer. Eur J Cancer 2019;106:45-53.

44 National Comprehensive Cancer Network. NCCN clinical practice guidelines in oncology (NCCN guidelines): genetic/familial highrisk assessment: breast and ovarian. version 1.2018. National Comprehensive Cancer Network, 2018.

45 US Preventive Services Task Force. Genetic risk assessment and BRCA mutation testing for breast and ovarian cancer susceptibility: recommendation statement. Ann Intern Med 2005;143:355-61.

46 Wetterstrand K. DNA sequencing costs: data from the NHGRI genome sequencing program (GSP). Rockville Pike, Bethesda, United States: National Human Genome Research Institute, National institute of Health, 2016.

47 Curran GM, Bauer M, Mittman B, et al. Effectiveness-implementation hybrid designs: combining elements of clinical effectiveness and implementation research to enhance public health impact. Med Care 2012;50:217-26.

48 Proctor EK, Powell BJ, McMillen JC. Implementation strategies: recommendations for specifying and reporting. Implement Sci 2013;8:139.

49 Kemp CG, Jarrett BA, Kwon C-S, et al. Implementation science and stigma reduction interventions in low- and middle-income countries: a systematic review. BMC Med 2019;17:6.

50 Hoffmann TC, Glasziou PP, Boutron I, et al. Better reporting of interventions: template for intervention description and replication (TIDieR) checklist and guide. BMJ 2014;348:g1687.

51 Kosztolányi G. 3. risk assessment. EJIFCC 2008;19:13-21.

52 Roberts MC, Kennedy AE, Chambers DA, et al. The current state of implementation science in genomic medicine: opportunities for improvement. Genet Med 2017;19:858-63.

53 Fulda KG, Lykens K. Ethical issues in predictive genetic testing: a public health perspective. J Med Ethics 2006;32:143-7. 